\title{
En-face optical coherence tomography angiography of neovascularization elsewhere in hemicentral retinal vein occlusion
}

This article was published in the following Dove Press journal:

International Medical Case Reports Journal

23 October 2015

Number of times this article has been viewed

\section{Kenji Sogawa}

Taiji Nagaoka

Akihiro Ishibazawa

Atsushi Takahashi

Tomofumi Tani

Akitoshi Yoshida

Department of Ophthalmology, Asahikawa Medical University,

Asahikawa, Japan
Correspondence: Kenji Sogawa Department of Ophthalmology, Asahikawa Medical University, Midorigaoka Higashi I-I, Asahikawa, Hokkaido 078-85I0, Japan

Tel +8I I66 682543

Fax +81166682549

Email cdh72740@yahoo.co.jp
Purpose: To evaluate how the growth of neovascularization elsewhere (NVE) was delineated in an eye with hemicentral retinal vein occlusion (CRVO) using optical coherence tomography (OCT) angiography.

Patients and methods: We examined a 64-year-old man diagnosed with hemi-CRVO. The area around the occluded vein was scanned using a spectral-domain OCT device (RTVue XR Avanti). Blood flow was detected using the split-spectrum amplitude-decorrelation angiography (SSADA) algorithm. Color fundus photography, fluorescein angiography (FA), and OCT angiography examinations were performed at the first visit and at 3 and 6 months postpresentation.

Results: At the first visit, FA revealed delayed retinal venous filling and extensive areas of capillary nonperfusion. The patient underwent a trial of intravitreal ranibizumab injection $(0.5 \mathrm{mg} / 0.05$ $\mathrm{mL}$ ) for the treatment of macular edema. At 3 months postpresentation, there was no NVE around the occluded vein in the en-face SSADA image, but at 6 months, NVE appeared on the occluded veins. The en-face SSADA image showed the NVE structure in the fibrovascular membrane on the occluded vein more clearly than FA images.

Conclusion: OCT angiography clearly visualized the sprouting of NVE in an eye with hemiCRVO. New findings of the vascular structure of NVE in hemi-CRVO were revealed using the en-face SSADA algorithm.

Keywords: OCT angiography, hemi-CRVO, NVE

\section{Introduction}

Central retinal vein occlusion (CRVO) is a cause of visual loss in developed countries. ${ }^{1,2}$ CRVO is divided into two types: nonischemic and ischemic CRVO. ${ }^{1,3}$ The visual prognosis for patients with ischemic CRVO is worse than that for patients with nonischemic CRVO. ${ }^{2,3}$ Patients with ischemic CRVO and branch retinal vein occlusion have a greater risk of developing neovascularization elsewhere (NVE), ${ }^{3}$ which can lead to vitreous hemorrhage and neovascular glaucoma, and result in loss of vision. ${ }^{3,4}$

Fluorescein angiography (FA) is a very important tool for evaluating nonperfusion areas and detecting neovascularization (NV) in ischemic CRVO. ${ }^{5}$ Although FA is vital for CRVO examination, intravenous fluorescein injection should be carefully performed because dye injection can occasionally cause nausea and, rarely, anaphylaxis. Furthermore, it is difficult to evaluate the detailed vasculature in NV by FA because of leakage of the dye toward the vitreous cavity.

Recent developments in theoretically-based optical coherence tomography (OCT) angiography allow for noninvasive evaluation of capillary vasculature. ${ }^{6-10}$ In particular, use of the split-spectrum amplitude-decorrelation angiography (SSADA) algorithm 
improves the signal-to-noise ratio of flow detection. ${ }^{8}$ The en-face SSADA images provide a clearer image of the vasculature than FA. Furthermore, the en-face SSADA images can help in the clear visualization of the microvasculature in different retinal layers. ${ }^{11}$ Some studies have evaluated choroidal NV using OCT angiography by applying the SSADA algorithm, ${ }^{7,12}$ and we previously reported that the NV structures could be clearly visualized at the disc in enface SSADA images obtained from patients with diabetic retinopathy. ${ }^{11}$ No studies to date have evaluated retinal NV in CRVO using en-face SSADA imaging.

In this study, we used OCT angiography with application of the SSADA algorithm to evaluate the microvasculature of NVE in ischemic hemi-CRVO.

\section{Patient and methods}

A 64-year-old man diagnosed with hemi-CRVO in his right eye was recruited. This patient was examined in the Department of Ophthalmology of Asahikawa Medical University. Best-corrected visual acuity (BCVA), slitlamp examination, fundus biomicroscopy, color fundus photograph, intraocular pressure (IOP), FA, OCT, and OCT angiography (vide infra) were evaluated at the first visit and at 3 and 6 months postpresentation. Central macular thickness was defined as the region of tissue between the inner limiting membrane and the outer retinal pigment epithelium boundary at the fovea for OCT images. The FA was performed with Spectralis HRA + OCT (Heidelberg Engineering, Heidelberg, Germany). This study was performed in adherence to the tenets of the Declaration of Helsinki and was approved by our institutional review board at the Asahikawa Medical University. Informed consent was obtained from the patient.

The instrument used for the OCT angiographic images was based on the RTVue XR Avanti (Optovue Inc., Fremont, CA, USA) and was used to obtain en-face SSADA images, as previously described. ${ }^{11}$ The scanning area was captured in $3 \times 3 \mathrm{~mm}^{2}$ sections and focused on the occluded vein near the optic disc head. The OCT angiograms were segmented as follows; the en-face image was segmented with an inner boundary set at $3 \mu \mathrm{m}$ beneath the internal limiting membrane and an outer boundary set at $15 \mu \mathrm{m}$ beneath the inner plexiform layer (IPL) to obtain images of the superficial vascular layers (defined as the superficial plexus), the en-face image was segmented with an inner boundary at $15 \mu \mathrm{m}$ beneath the IPL and the outer boundary was set at $70 \mu \mathrm{m}$ beneath the IPL to obtain images of the deep vascular layers (defined as the deep plexus).

\section{Results}

At the first visit, BCVA in the right eye was 20/100 and IOP was $14 \mathrm{mmHg}$. Fundus examination revealed numerous retinal hemorrhages in half of the inferior part, dilated and tortuous retinal veins, macular edema, and cotton wool spots (Figure 1A). The left eye had a completely normal fundus. The central macular thickness in his right eye was $721 \mu \mathrm{m}$, indicating severe macular edema. FA revealed delayed retinal venous filling and extensive areas of capillary nonperfusion (Figure 1B). The patient was diagnosed with ischemic hemiCRVO and underwent intravitreal injection of ranibizumab $(0.5 \mathrm{mg} / 0.05 \mathrm{~mL})$ for the treatment of macular edema.

One month after the first visit, his BCVA had improved to 20/25 and central macular thickness decreased significantly to $296 \mu \mathrm{m}$. At 3 months postpresentation, his BCVA had further improved to 20/20. Fundus examination revealed a reduction in the retinal hemorrhage, cotton wool spots, and macular edema $(256 \mu \mathrm{m})$. At 6 months after the first visit, there was no macular edema. His BCVA was 20/20, and his IOP was $15 \mathrm{mmHg}$. Fundus examination revealed almost no change from the appearance at 3 months (Figure 1C). FA, however, revealed an extensive nonperfused area and emergence of NVE at the occluded inferior temporal vein (Figure 1D).

In the en-face SSADA images of the superficial plexus, there was no NVE around the occluded vessel (Figure 2A) at 3 months postpresentation, but at 6 months, remarkable NVE growth was observed on the occluded vein (Figure 2B). NVE was not visualized in the en-face SSADA image of the deep plexus (Figure 2C). The en-face SSADA image more clearly

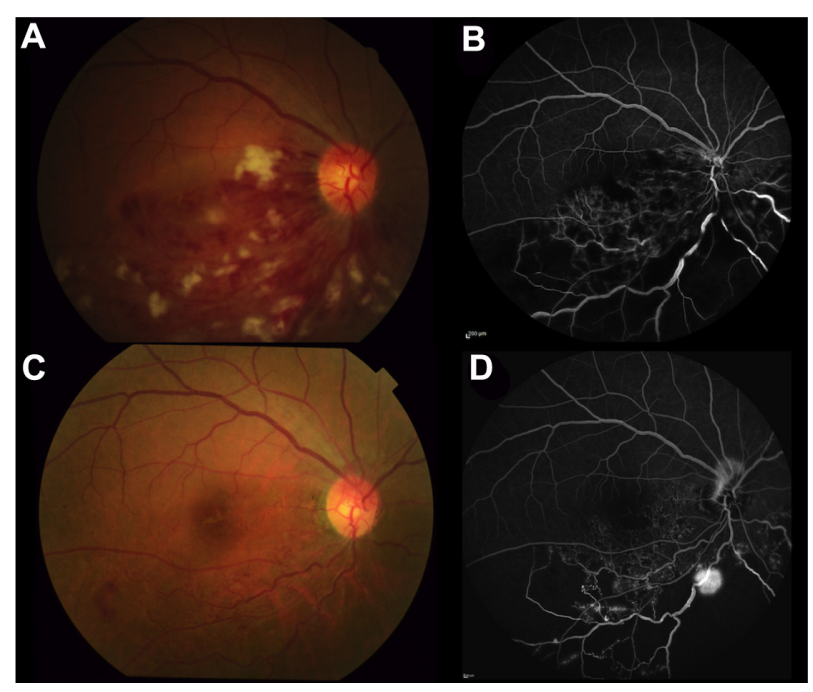

Figure I Color fundus photography and FA at the first visit (A and B) and at 6 months postpresentation ( $\mathbf{C}$ and $\mathbf{D}$ ).

Abbreviation: FA, fluorescein angiography. 


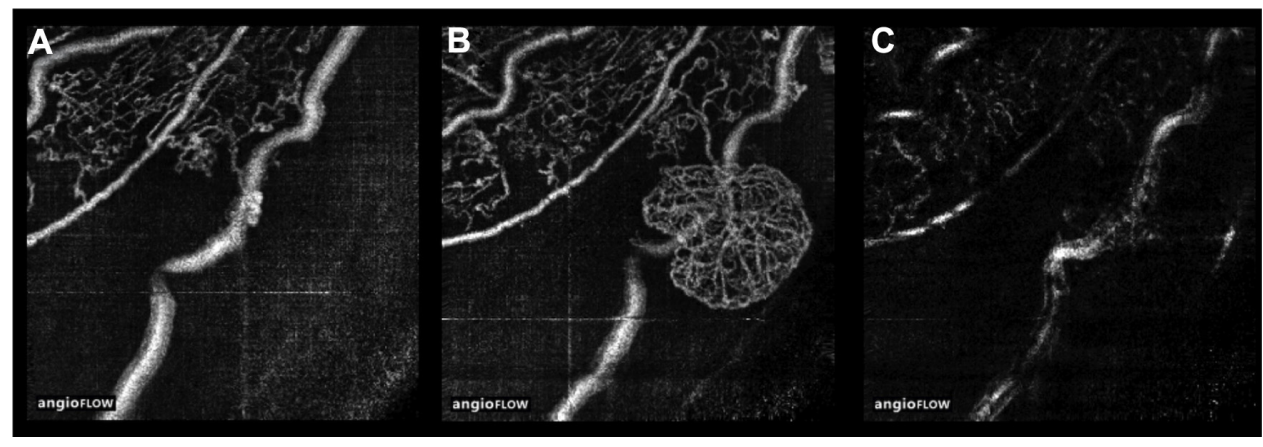

Figure 2 En-face SSADA image of the superficial plexus at 3 months (A) and 6 months (B) postpresentation. NVE appeared around the occluded veins. (C) The deep plexus image at 6 months postpresentation.

Notes: NVE was not visualized in this image.

Abbreviations: SSADA, split-spectrum amplitude-decorrelation angiography; NVE, neovascularization elsewhere.

revealed the NVE structure in the fibrovascular membrane (Figure 2B) than the FA image (Figure 1D).

\section{Discussion}

This is the first study to show the growth of NVE in a hemi-CRVO eye using en-face SSADA imaging. Although NVE can also be evaluated by FA examination, the vascular structure of NVE cannot be clearly visualized by FA because of excessive leakage from NVE, even in the early phase of FA. The current study demonstrated that en-face SSADA imaging, which is not affected by leakage, clearly visualizes the growth and detailed microvasculature of NVE in hemi-CRVO.

Some studies have described NV at the disc in diabetic retinopathy using spectral-domain OCT. ${ }^{13,14}$ Few studies, however, have examined NVE. ${ }^{9,13}$ Cho et al ${ }^{13}$ reported that a patient with diabetic retinopathy and NVE had a thickened posterior hyaloid, and NVE was observed as hyperreflective material within the inner retinal layer and as projecting in spectral-OCT images. Moreover, in a recent Doppler OCT study, Miura et $\mathrm{al}^{9}$ evaluated NVE in diabetic retinopathy. In this study, we observed that the posterior hyaloid rigidly adhered at the point of the NVE in the hemi-CRVO eye in

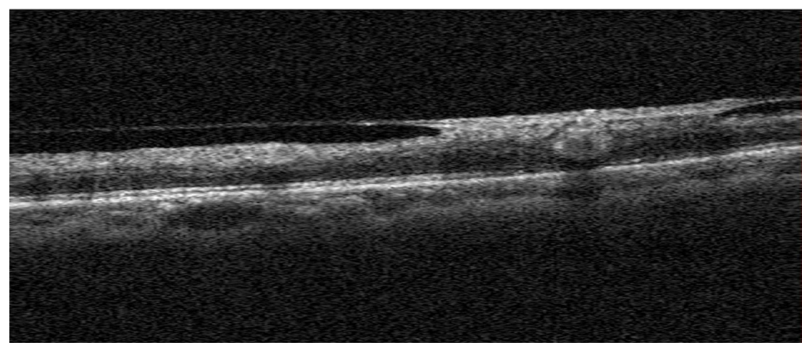

Figure 3 OCT B-scan image of NVE.

Note: The posterior hyaloid rigidly adhered at the point of the NVE.

Abbreviations: OCT, optical coherence tomography; NVE, neovascularization elsewhere.
OCT B-scan images using OCT angiography (Figure 3). This finding is quite similar to NVE in diabetic retinopathy.

The findings of this study also elucidated that NVE of hemi-CRVO was located only in the superficial retinal layer because there was no vascular structure of NVE in the enface SSADA image of the deep plexus (Figure 2B and C). The origin of NVE vessels in hemi-CRVO may be the radial peripapillary capillaries because 3 months before the growth of NVE we observed the growth of radial peripapillary capillaries into the occluded vein at the same point of the growth of NVE. Furthermore, the NVE of hemi-CRVO was round in shape, and the outer border of the NVE terminus of the vessels created loops. Spaide ${ }^{12}$ reported that in some lesions of choroidal NV, the outer terminus was composed of vascular loops. This feature of NV is similar to what we observed. Taken together, the NVE in hemi-CRVO, comprising looping radial peripapillary capillaries, sprouted on the occluded vein in the superficial retinal layer.

This study has some limitations. First, this study was a case study. A large number of patients are needed to evaluate the significant features of NVE of hemi-CRVO. Second, we have not evaluated the growth processes of NVE in hemiCRVO. In this patient, we performed photocoagulation in the area of nonperfusion. It would be interesting to determine whether the NVE might eventually disappear using en-face SSADA images.

\section{Conclusion}

This study demonstrates that NVE sprouting can be observed in hemi-CRVO by en-face SSADA imaging.

\section{Disclosure}

The authors report no conflicts of interest in this work. 


\section{References}

1. Bhagat N, Goldberg MF, Gascon P, Bell W, Haberman J, Zarbin MA. Central retinal vein occlusion: review of management. Eur J Ophthalmol. 1999;9(3):165-180.

2. Hayreh SS. Management of central retinal vein occlusion. Ophthalmologica. 2003;217(3):167-188.

3. Rehak M, Wiedemann P. Retinal vein thrombosis: pathogenesis and management. J Thromb Haemost. 2010;8(9):1886-1894.

4. Chan CK, Ip MS, Vanveldhuisen PC, et al. SCORE Study report \#11: incidences of neovascular events in eyes with retinal vein occlusion. Ophthalmology. 2011;118(7):1364-1372.

5. Hayreh SS. Retinal vein occlusion. Indian J Ophthalmol. 1994;42(3): 109-132.

6. Huang Y, Zhang Q, Thorell MR, et al. Swept-source OCT angiography of the retinal vasculature using intensity differentiation-based optical microangiography algorithms. Ophthalmic Surg Lasers Imaging Retina. 2014;45(5):382-389.

7. Jia Y, Bailey ST, Wilson DJ, et al. Quantitative optical coherence tomography angiography of choroidal neovascularization in age-related macular degeneration. Ophthalmology. 2014;121(7):1435-1444.

8. Jia Y, Tan O, Tokayer J, et al. Split-spectrum amplitude-decorrelation angiography with optical coherence tomography. Opt Express. 2012; 20(4):4710-4725.
9. Miura M, Hong YJ, Yasuno Y, Muramatsu D, Iwasaki T, Goto H. Three-dimensional vascular imaging of proliferative diabetic retinopathy by Doppler optical coherence tomography. Am J Ophthalmol. 2015;159(3):528-538. e523.

10. Spaide RF, Klancnik JM Jr, Cooney MJ. Retinal vascular layers imaged by fluorescein angiography and optical coherence tomography angiography. JAMA Ophthalmol. 2015;133(1):45-50.

11. Ishibazawa A, Nagaoka T, Takahashi A, et al. Optical coherence tomography angiography in diabetic retinopathy: a prospective pilot study. Am J Ophthalmol. 2015;160(1):35-44.

12. Spaide RF. Optical coherence tomography angiography signs of vascular abnormalization with antiangiogenic therapy for choroidal neovascularization. Am J Ophthalmol. 2015;160(1):6-16.

13. Cho H, Alwassia AA, Regiatieri CV, et al. Retinal neovascularization secondary to proliferative diabetic retinopathy characterized by spectral domain optical coherence tomography. Retina. 2013;33(3):542-547.

14. Muqit MM, Stanga PE. Fourier-domain optical coherence tomography evaluation of retinal and optic nerve head neovascularisation in proliferative diabetic retinopathy. Br J Ophthalmol. 2014;98(1):65-72.
International Medical Case Reports Journal

\section{Publish your work in this journal}

The International Medical Case Reports Journal is an international, peer-reviewed open-access journal publishing original case reports from all medical specialties. Previously unpublished medical posters are also accepted relating to any area of clinical or preclinical science. Submissions should not normally exceed 2,000 words or

\section{Dovepress}

4 published pages including figures, diagrams and references. The manuscript management system is completely online and includes a very quick and fair peer-review system, which is all easy to use. Visit $\mathrm{http}: / /$ www.dovepress.com/testimonials.php to read real quotes from published authors. 